\title{
ANÁLISIS EXPLORATORIO DE DATOS ESPACIALES ESTADÍSTICA ESPACIAL
}

\section{DEPARTAMENTO DE GEOGRAFIA FACULTAD DE HUMANIDADES $\bullet$ UNNE Prof. Silvia Stela Ferreyra}

Publicado en formato digital: Prof. Silvia Stela FERREYRA. ANÁLISIS EXPLORATORIO DE DATOS ESPACIALES. Estadística Espacial. Resúmenes. Revista Geográfica Digital. IGUNNE. Facultad de Humanidades. UNNE, Año 11. No 21. Enero - Junio 2014. ISSN 1668-5180. Resistencia, Chaco. En: http:/hum.unne.edu.ar/revistas/geoweb/default.htm 
serie de datos numéricos presentados de manera ordenaday sistemática,

\section{Estadística:}

pero cuando comenzamosa adentrarnos en la investigación, empezamosa percibir a la estadística como una herramienta para obtener resultados confiables en una Investigación.
La Investigación puede tener como objetivos:

- El análisis exploratorio de datos que va más allá de una descripción de los mismos sino que es parte de la actitud que define al buen investigador, es decir aprender a observar que expresan esos datos, como ser encontrar patrones de comportamiento o anomalías en las distribuciones.

- El análisis confirmatorio de datos relacionado con la estadística inferencial, que comienza con la formulación de hipótesis que luego serán confrontadas con los datos empíricos por medio de metodologías estadísticas adecuadas. 
“El Análisis de Datos" o Estadística descriptiva comprende:

Serie de técnicas orientadas a extraer información de un conjunto de datos, mediante medidas que resumen los principales rasgos de la distribución de frecuencias de ese conjunto de datos. 

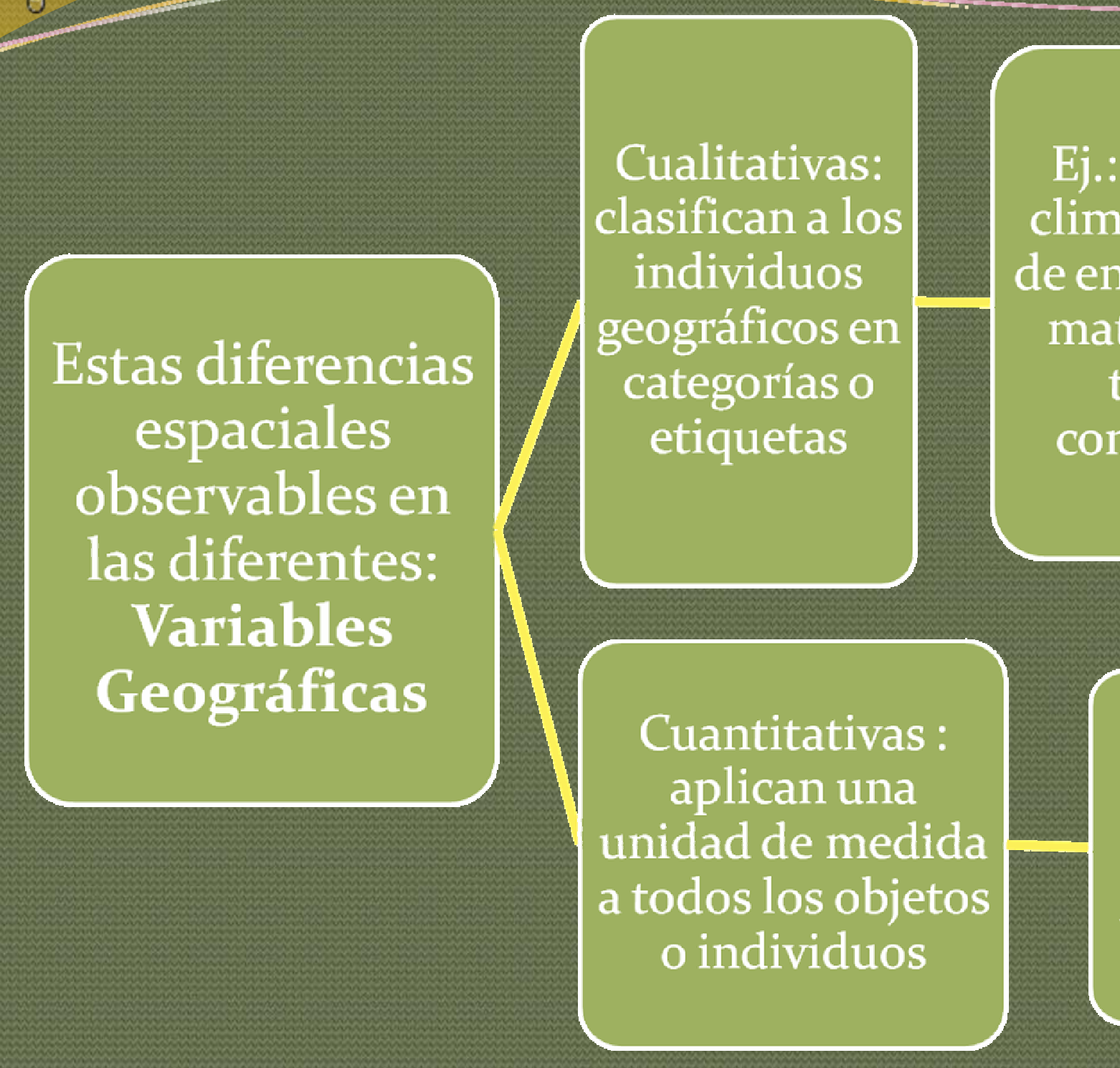

Ej.: uso de suelos, tipo de clima, tipo de relieve, tipos de enfermedades, calidad de materiales de la vivienda, tipo de instrucción, condición ocupacional... observables en las diferentes: Variables Geográficas

Publicado en formato digital: Prof. Silvia Stela FERREYRA. ANÁISIS EXPLORATORIO DE DATOS ESPACIALES. Estadística ESpacial. Resúmenes. Revista Geográfica Digital. IGUNNE. Facultad de Humanidades. UNNE. Año 11. № 21. Enero-Junio 2014. ISSN 1668-5180. Resistencia, Chaco. En:

Ej.: NBI, Tasa de Natalidad, Mortalidad, Îndice de Masculinidad, Variación Intercensal, ... 
Cuando se dispone de una cantidad considerable de datos es útil presentarlos en:

\section{TIABLASS ESTI'ADISTIICAS}

- Permiten conocer el número de veces que se repite un fenómeno. En su estado más desagregado son listados de valores, que muestran la distribución de los valores de la variable.

- Con el fin de reducir el listado de valores, se agrupan los valores en clases y se presenta la frecuencia de las mismas, a estas tablas se denominan Tablas de Frecuencias.

-Es común presentar estas frecuencias absolutas como frecuencias relativas o porcentuales, y también puede presentarse en forma acumulada.

- Para establecer las clases o grupos que estará en función del número de observaciones, algunos autores proponen que una distribución debe tener un número de clases no inferior a 6 y no superior a 20. Según Huntesberge lo estima con $\mathrm{K}=1+3,3 \cdot \log \mathrm{n}$, siendo n el núm. total de observaciones.

-Rango = Vmax - Vmin

-C(ancho de clase)=Rango/ núm. de clases 


\section{Para datos sin agrupar}

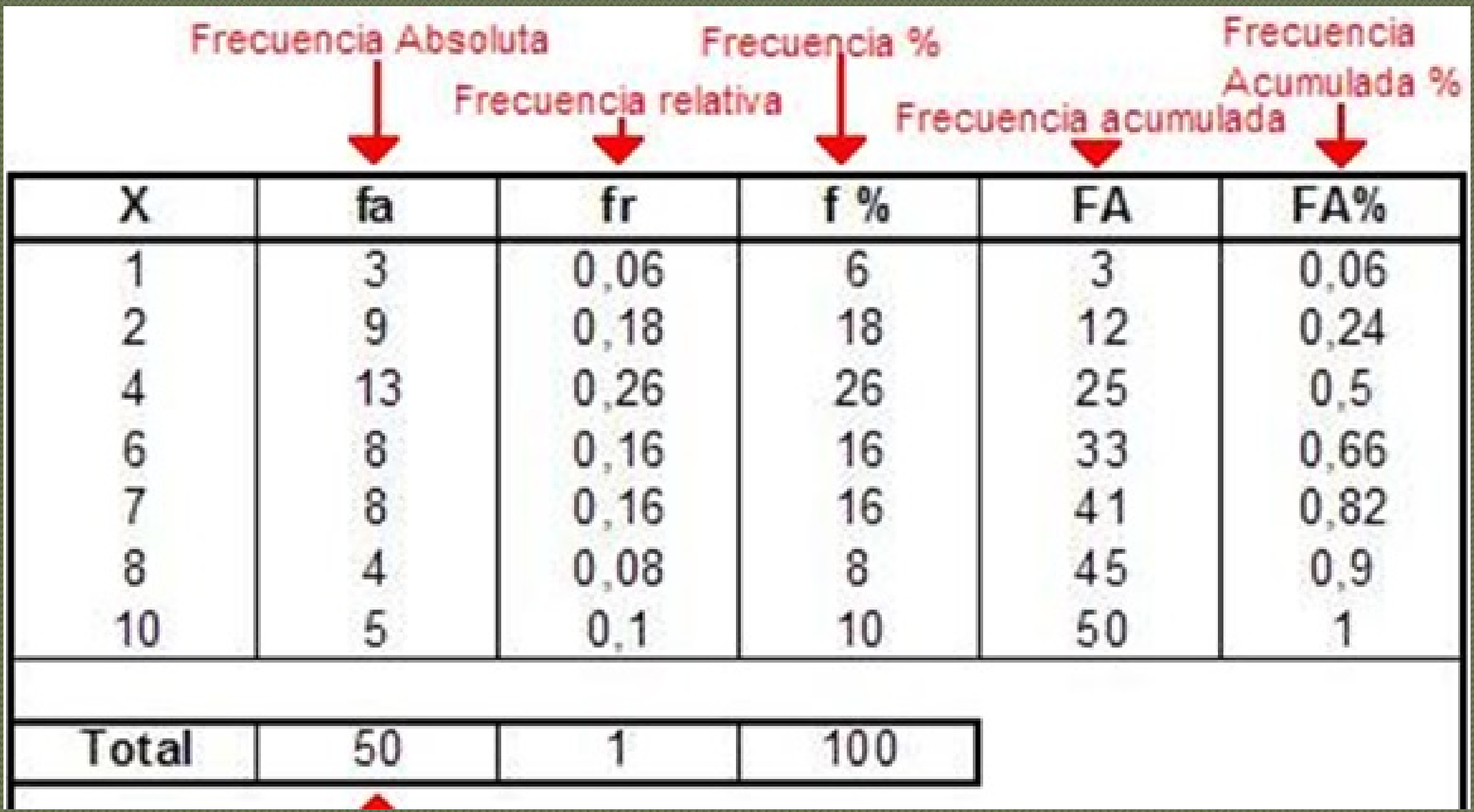

Publicado en formato digital: Prof. Silvia Stela FERREYRA. ANÁLISIS EXPLORATORIO DE DATOS ESPACIALES. Estadística Espacial. Resúmenes. Revista Geográfica Digital. IGUNNE. Facultad de Humanidades. UNNE. Año 11. № 21. Enero - Junio 2014. ISSN 1668-5180. Resistencia, Chaco. En: http:/hum.unne.edu.ar/revistas/geoweb/default.htm 


\section{SERIES TEMPORALES}

Distribución de una variable en el tiempo:

Para la descripción de una serie temporal es frecuente la elaboración de números índices(resultado de una comparación por cociente), y tomando el valor de un año base como el 100\%, se analiza la evolución por comparación.

$$
\begin{gathered}
\mathrm{I}(\mathrm{i} / \mathrm{t})=(\mathrm{Xi} / \mathrm{Xt})^{*} 100 \\
>100 \% \text { crecimiento } \\
<100 \% \text { decrecimiento }
\end{gathered}
$$

Publicado en formato digital: Prof. Silvia Stela FERREYRA. ANÁLISIS EXPLORATORIO DE DATOS ESPACIALES. Estadística Espacial. Resúmenes. Revista Geográfica Digital. IGUNNE. Facultad de Humanidades. UNNE. Año 11. № 21. Enero - Junio 2014. ISSN 1668-5180. Resistencia, Chaco. En: 
Revista Geográfica Digital. I GUNNE. Facultad de Humanidades. UNNE. Año 11. № 21. Enero - J unio 2014. ISSN 1668-5180 Resistencia, Chaco

\begin{tabular}{|c|c|c|c|c|c|}
\hline $\begin{array}{l}\text { CONC } \\
\text { Ej.: Estudia } \\
\text { en España } \\
\text { 1997, dete }\end{array}$ & $\begin{array}{r}\text { 2. NÚ } \\
\text { CEPTO Y P } \\
\underline{\mathbf{N}} \\
\text { r la evoluci } \\
\text { provenient } \\
\text { rminando lo }\end{array}$ & $\begin{array}{l}\text { IMEROS ÍN } \\
\text { ROPIEDAD } \\
\text { ÚMERO ÍNI } \\
\text { ón del númere } \\
\text { es de Franci } \\
\text { s correspondi }\end{array}$ & $\begin{array}{l}\text { SIMF } \\
\text { IDICE } \\
\text { IMPL } \\
\text { stas (e } \\
\text { ladá e } \\
\text { dices. }\end{array}$ & $\begin{array}{l}\text { LES: } \\
\text { S EN C } \\
\text { E } \\
\text { n miles) } \\
\text { ntre los }\end{array}$ & $\begin{array}{l}\text { ADENA } \\
\text { que ent } \\
\text { años } 19\end{array}$ \\
\hline Años & \begin{tabular}{|c} 
Turistas \\
Franceses
\end{tabular} & $\begin{array}{c}\text { Turistas } \\
\text { Canadienses }\end{array}$ & Años & $\mathrm{I}_{\mathrm{t} / 92}{ }^{\mathrm{F}}$ & $\mathrm{I}_{\mathrm{t} / 92}{ }^{\mathrm{C}}$ \\
\hline 1992 & 1035 & 44 & 1992 & 1 & 1 \\
\hline 1993 & 1230 & 56 & 1993 & 1,1884 & 1,2727 \\
\hline 1994 & 1351 & 65 & 1994 & 1,3053 & 1,4772 \\
\hline 1995 & 1230 & 85 & 1995 & 1,1884 & 1,9318 \\
\hline 1996 & 1750 & 116 & 1996 & 1,6908 & 2,6363 \\
\hline 1997 & 2554 & 153 & 1997 & 2,4676 & 3,4772 \\
\hline
\end{tabular}

\section{NÚMEROS ÍNDICES SIMPLES:} NÚMERO ÍNDICE SIMPLE

Ej.: Estudiar la evolución del número de turistas (en miles) que entraron en España provenientes de Francia y Canadá entre los años 1992 y 997, determinando los correspondientes índices.

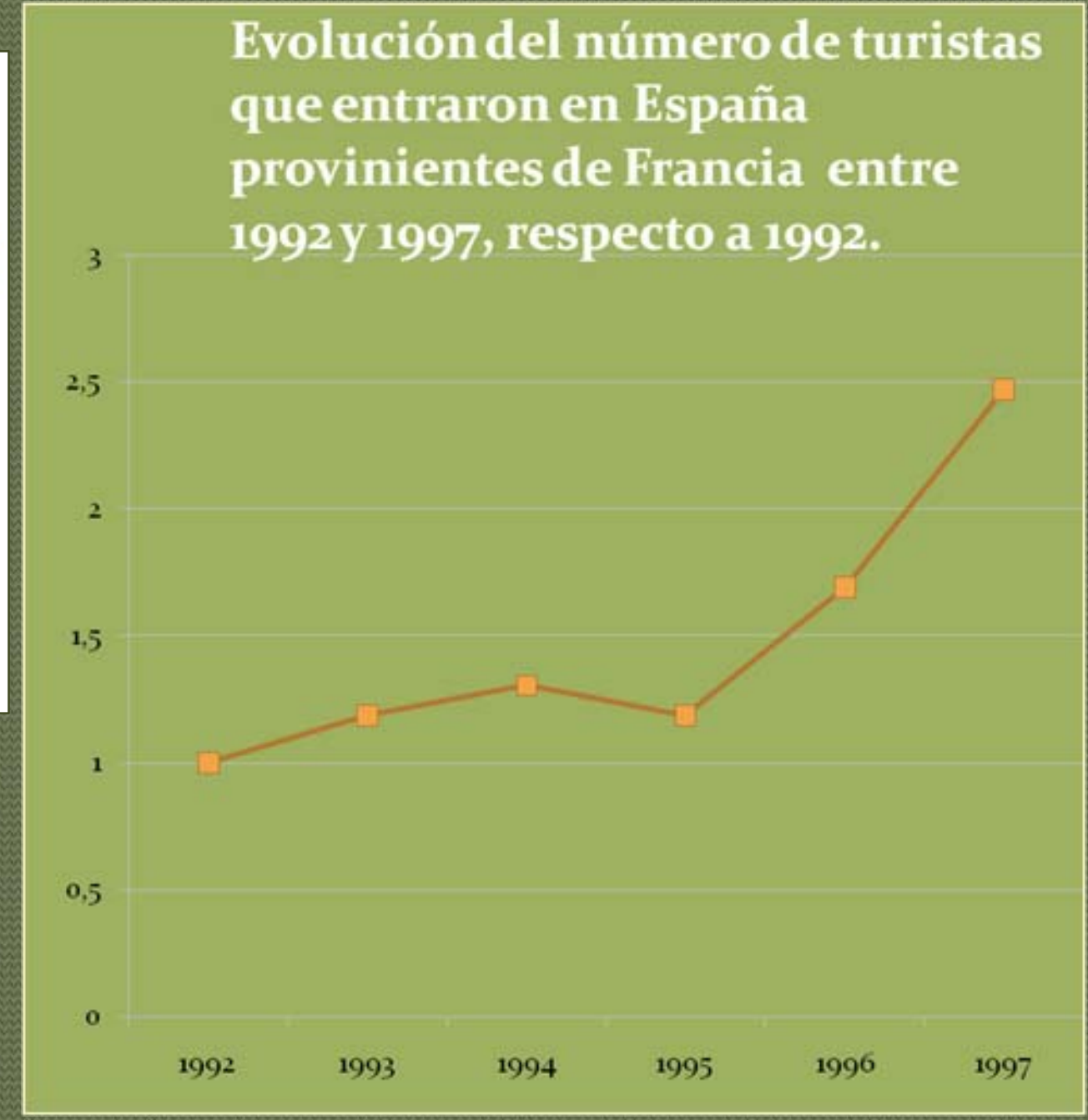

Publicado en formato digital: Prof. Silvia Stela FERREYRA. ANÁLISIS EXPLORATORIO DE DATOS ESPACIALES. Estadística ESPacial. Resúmenes. Revista Geográfica Digital. IGUNNE. Facultad de Humanidades. UNNE. Año 11. No 21. Enero-Junio 2014. ISSN 1668-5180. Resistencia, Chaco. En: http:l/hum.unne.edu.ar/revistas/geoweb/default.htm 
Revista Geográfica Digital. I GUNNE. Facultad de Humanidades. UNNE. Año 11. № 21. Enero - J unio 2014. ISSN 1668-5180 Resistencia, Chaco

\section{Los gráficos de series temporales, representan en el eje horizontal la variable temporal, mientras en el eje vertical los valores de la variable o la conversión en índices.).}

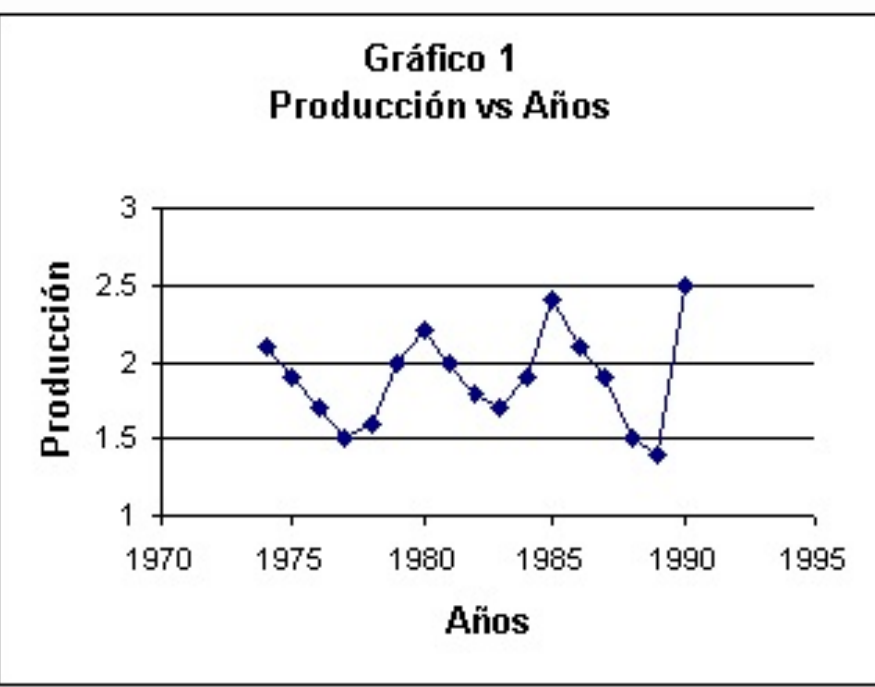

Producción de motocicletas en millones

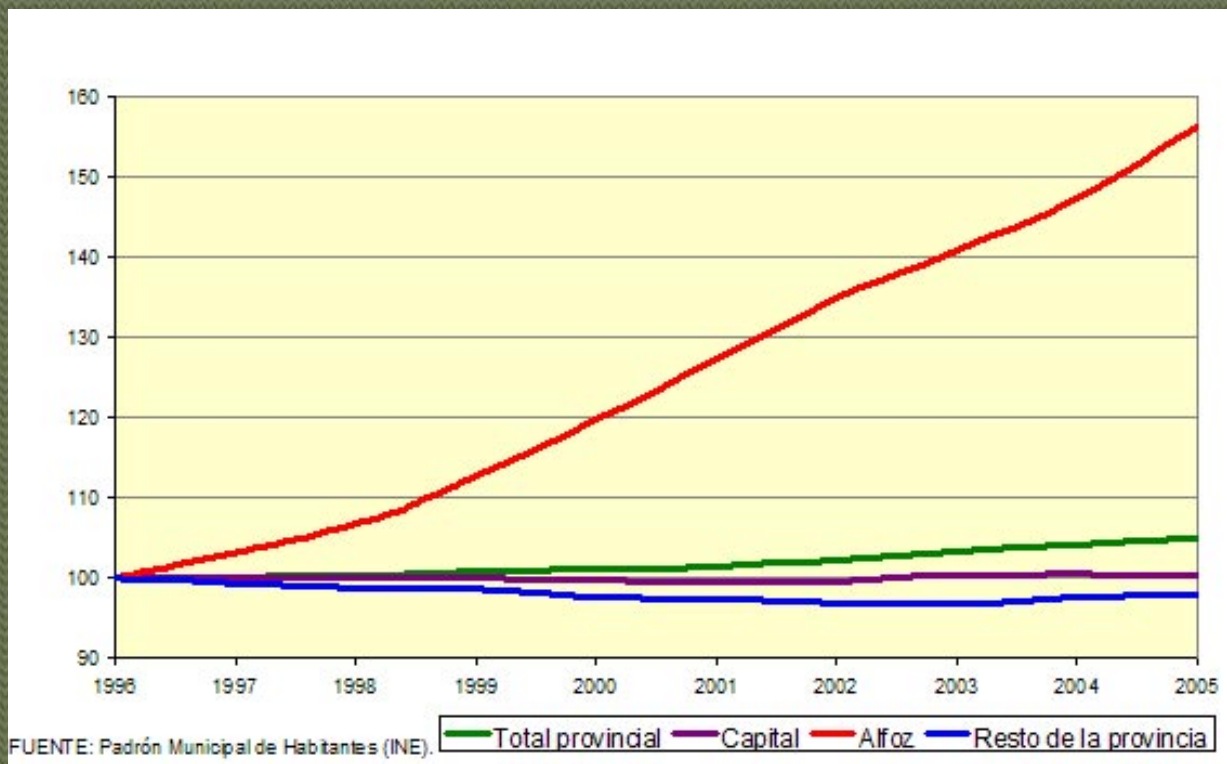

Evolución de la población de Valladolid, de su alfoz (entorno) y del resto de la provincia 1996-2005.

Números índice (1996=100

Publicado en formato digital: Prof. Silvia Stela FERREYRA. ANÁLISIS EXPLORATORIO DE DATOS ESPACIALES. Estadística ESPacial. Resúmenes. Revista Geográfica Digital. IGUNNE. Facultad de Humanidades. UNNE. Año 11. № 21. Enero - Junio 2014. ISSN 1668-5180. Resistencia, Chaco. En:

http:llhum.unne.edu.ar/revistas/geoweb/default.htm 


\section{REPRESENTACIONES GRAFICAS}

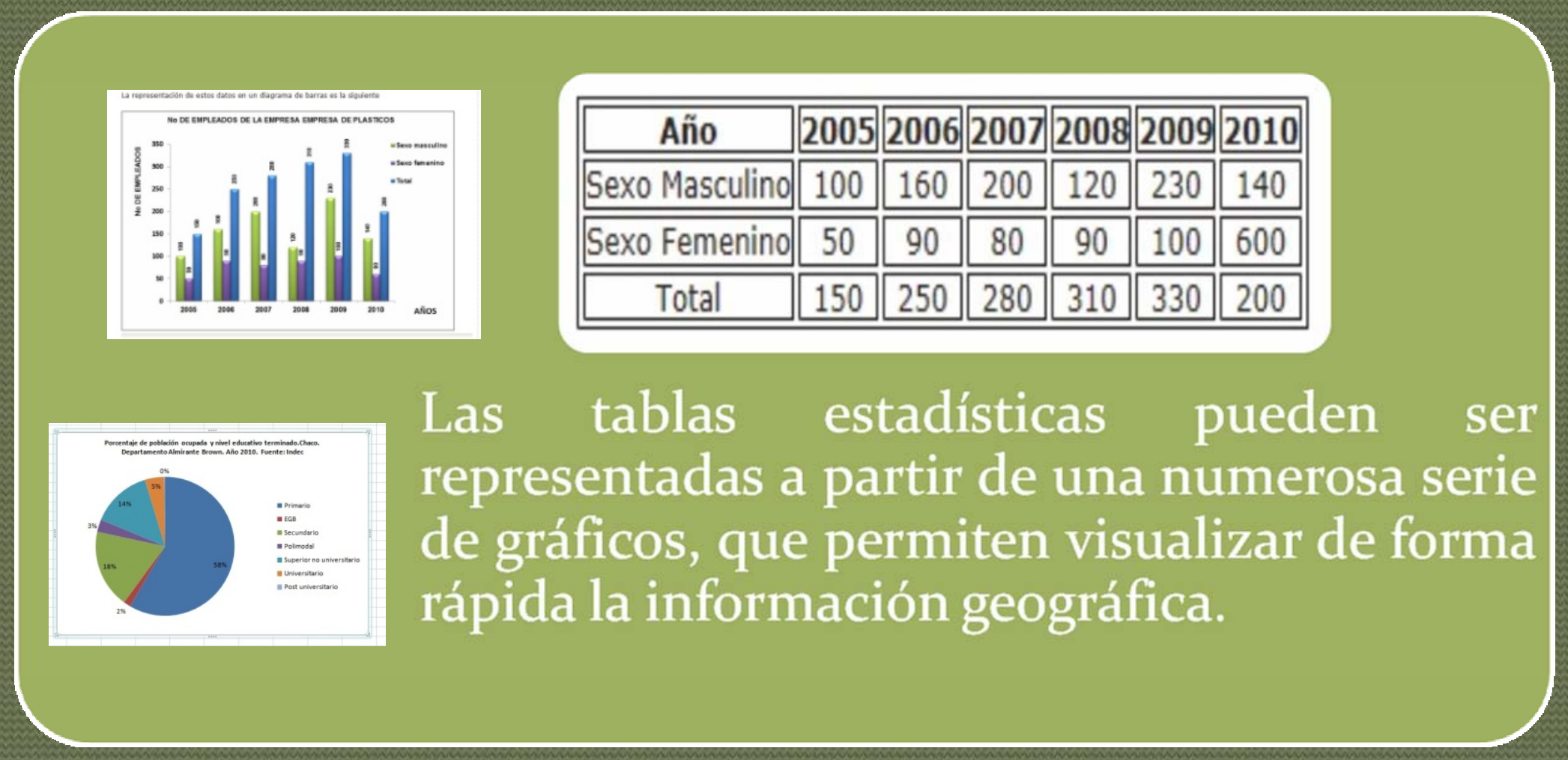

Publicado en formato digital: Prof. Silvia Stela FERREYRA. ANÁLISIS EXPLORATORIO DE DATOS ESPACIALES. Estadística ESPacial. Resúmenes. Revista Geográfica Digital. IGUNNE. Facultad de Humanidades. UNNE. Año 11. No 21. Enero - Junio 2014. ISSN 1668-5180. Resistencia, Chaco. En:

http:Ihum.unne.edu.ar/revistas/geoweb/default.htm 


\section{Diagrama de barras: permite representar la importancia cuantitativa de las diferentes categorías de una variable cualitativa o cuantitativa, por ejemplo: distribución de usos de suelos en un determinado espacio.}

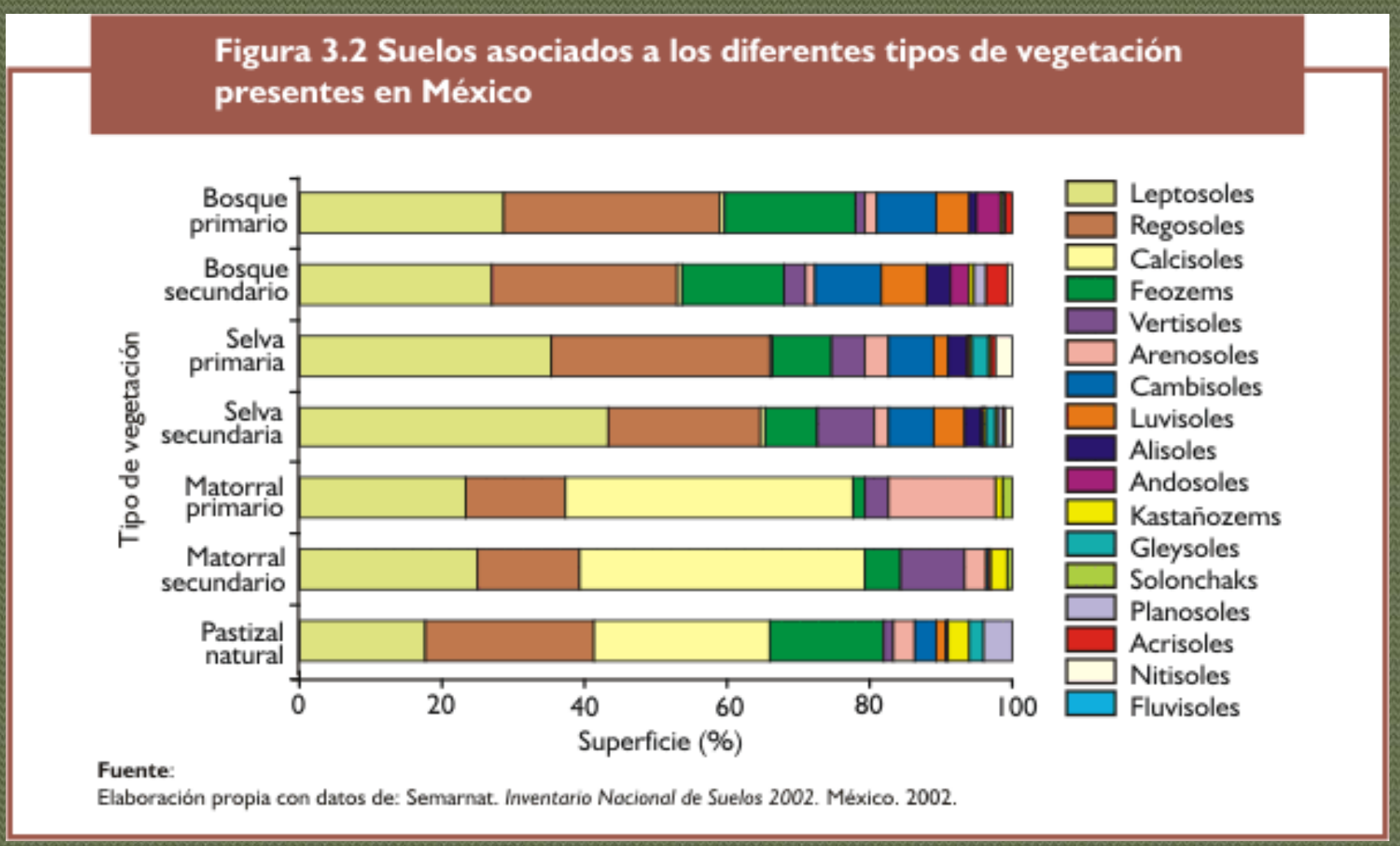


El histograma de frecuencias se construye sobre un eje vertical, que representa la frecuencia absoluta o relativa y en el eje horizontal van las clases definidas por su intervalo y punto medio o marca de clase, que sirven para trazar el polígono de frecuencias.

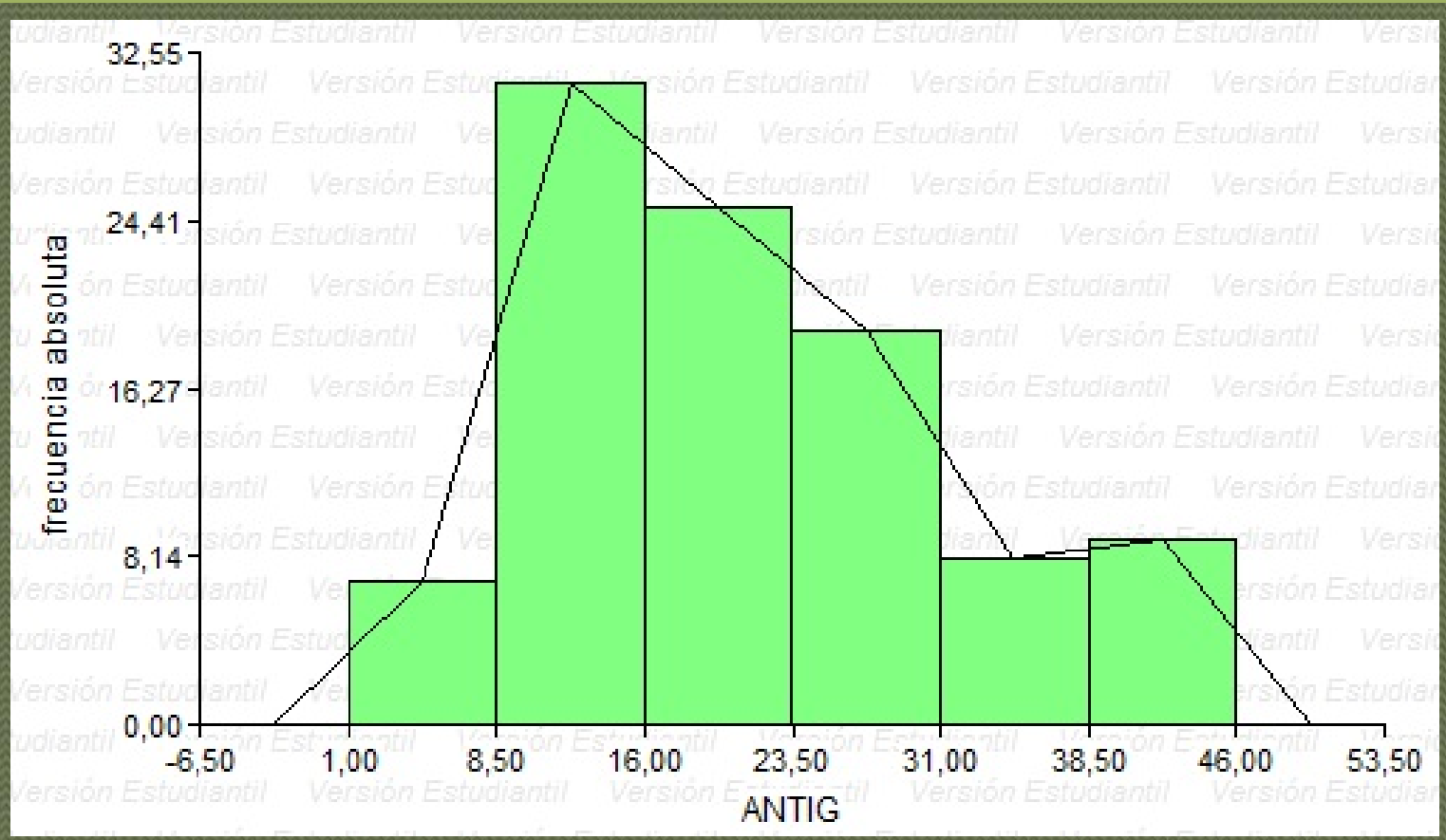

Publicado en formato digital: Prof. Silvia Stela FERREYRA. ANALISIS EXPLORATORIO DE DATOS ESPACIALES. Estadística Espacial: Resúmenes. Revista Geográfica Digital. IGUNNE. Facultad de Humanidades. UNNE. Año 11. No 21. Enero - Junio 2014. ISSN 1668-5180. Resistencia, Chaco. En: http:l/hum.unne.edu.ar/revistas/geoweb/default.htm 


\section{Diagrama de sectores puede usarse indistintamente para variables cualitativas o cuantitativas, hace equivaler la frecuencia de la categoría a un área proporcional del círculo.}

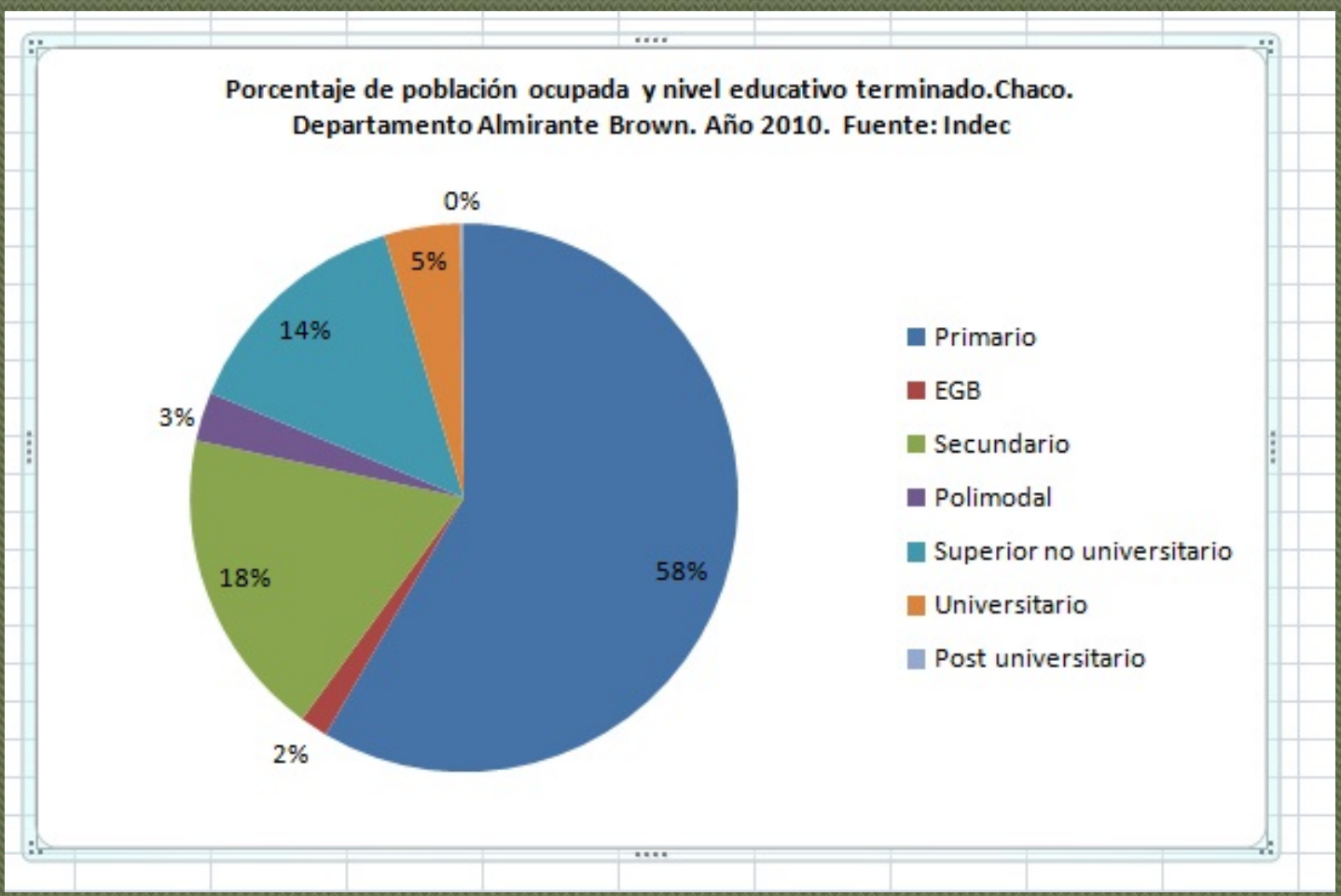

Publicado en formato digital: Prof. Silvia Stela FERREYRA. ANÁLISIS EXPLORATORIO DE DATOS ESPACIALES. Estadística Espacial. Resúmenes. Revista Geográfica Digital. IGUNNE. Facultad de Humanidades. UNNE. Año 11. № 21. Enero - Junio 2014. ISSN 1668-5180. Resistencia, Chaco. En:

http:Ihum.unne.edu.ar/revistas/geoweb/default.htm 


\section{MEDIDAS DESCRIPTIVAS}

\section{Medidas de tendencia central:}

\section{5}

1) Media Aritmética: la más conocida, sumatoria de todos los valores de la variable dividido por el número de datos.

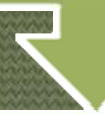

$\mathrm{Xmedia}=\sum \mathrm{X}_{\mathrm{i} / \mathrm{N} \text {, para datos sin agrupar }}$

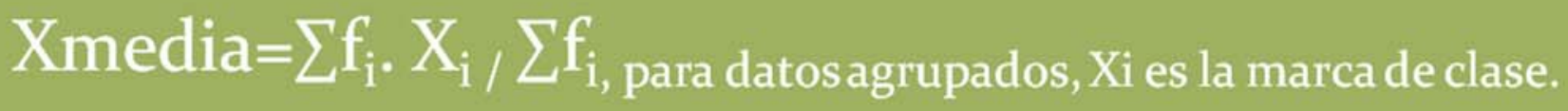

Xmedia ponderada $=\sum \mathrm{X}_{\mathrm{i}}, \mathrm{W}_{\mathrm{i} /} \sum \mathrm{W}_{\mathrm{i}}$

silos datosestán aquiupados Xies la marca declase. 
Xmedia Armónica $=1 / \sum 1 / \mathrm{X}_{\mathrm{i}}$, cuando se trabaja con variables referidasa tasas de cambio o movimiento

La media armónica se define como el recíproco de la media aritmética de los recíprocos:

Este valor se emplea para promediar variaciones con respecto al tiempo.

\section{Xmedia Geométrica $=\sum \log \mathrm{X}_{\mathrm{i}} / \mathrm{N}$, cuando la variable crece de forma geométrica}

Existen dos usos principales de la media geométrica:

Para promediar porcentajes, índices y cifras relativas y

Para determinar el incremento porcentual promedio en

ventas, producción u otras actividades o series económicas de un periodo a otro. La media geométrica no se ve tan afectada por valores extremos.

Publicado en formato digital: Prof. Silvia Stela FERREYRA. ANALISIS EXPLORATORIO DE DATOS ESPACIALES. Estadistica Espacial. Resúmenes. Revista Geográfica Digital. IGUNNE. Facultad de Humanidades. UNNE. Año 11. № 21. Enero - Junio 2014. ISSN 1668-5180. Resistencia, Chaco. En:

http:l/hum.unne.edu.ar/revistas/geoweb/default.htm 


\section{MEDIDAS DE TENDENCIA CENTRAL}

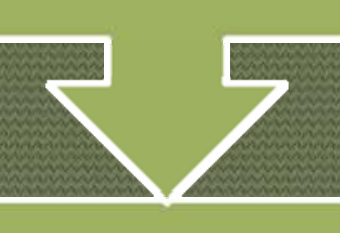

2) Moda: es el valor más observado o que más se repite, puede haber más de un a moda

Moda=Li + c. $($ di $/$ di. d2 $)$, para datos agrupados. 


\section{MEDIDAS DE TENDENCIA CENTRAL}

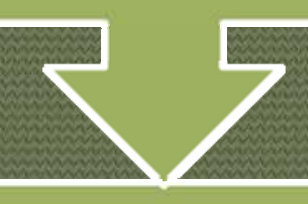

3) Mediana: es el valor tal que ordenados los datos de menor a mayor, deja por debajo y por arriba el $50 \%$ de los datos de la distribución.

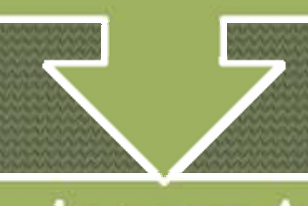

Mediana=Li + c.(N/2-facum_interv.Anteriores, fintMna), para datos agrupados.

Li: límite real inferior del intervalo que contiene a la mediana. C amplitud del intervalo 
1) Cuartiles: se calculan ordenando los datos de menor a mayor y se buscan los tres valores que dividen al conjunto de datos en cuatro partes de igual cantidad de datos. Entre $C_{1}$ y $C_{3}$ se encuentra el 50\% de los datos.

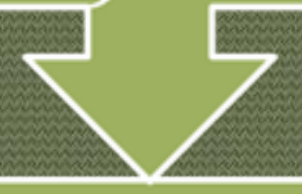

$\mathrm{C}_{1}$ : el 25\% de los datos son menores al valor de $\mathrm{C}_{1}$.

$\mathrm{C} 1=\mathrm{Li}+\mathrm{c} .(\mathrm{N} / 4$-facum_interv.Anteriores, fintC1), para datosagrupados.

Li: límite real inferior del intervalo que contiene al primer cuartil. C amplitud del intervalo

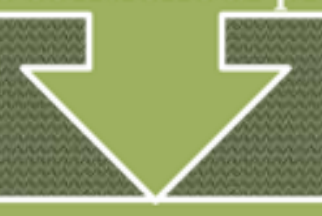

$\mathrm{C}_{2}$ : el $50 \%$ de los datos son menores al valor de $\mathrm{C}_{2}$.

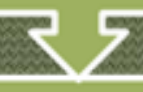

$\mathrm{C}_{3}$ : el $75 \%$ de los datos son menores al valor de $C_{3}$. 


\section{MEDIDAS DE POSICIÓN NO CENTRALES}

2) Quintiles: se calculan ordenando los datos de menor a mayor y se buscan los cuatro valores que dividen al conjunto de datos en cinco partes de igual cantidad de datos.

\section{Q1: el 20\% de los datos son menores al valor de Q1.}

$\mathrm{Q}_{1}=\mathrm{Li}+\mathrm{c}$.(N/5-facum_interv.Anteriores/fint $\left.\mathrm{Q}_{1}\right)$, para datos agrupados.

Li: límite real inferior del intervaloque contieneal primer quintil. C amplitud del intervalo

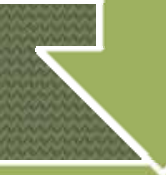

Q2: el 40\% de los datos son menores al valor de Q2.

证

Q3: el 60\% de los datos son menores al valor de $Q_{3}$.

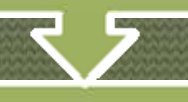

$\mathrm{Q}_{4}$ : el 80\% de los datos son menores al valor de $\mathrm{Q}_{4}$. 


\section{MEDIDAS DE POSICIÓN NO CENTRALES}

3) Deciles: se calculan ordenando los datos de menor a mayor y se buscan los nueve valores que dividen al conjunto de datos en diez partes de igual cantidad de datos, ( $\left.\mathrm{D}_{1}, \mathrm{D}_{2}, \mathrm{D}_{3}, \ldots . ., \mathrm{D}_{9}\right)$.

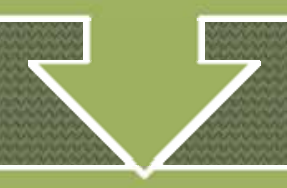

4) Percentiles: se calculan ordenando los datos de menor a mayor y se buscan los noventa y nueve valores que dividen al conjunto de datos en cien partes de igual cantidad de datos, $\left(\mathrm{P}_{1}, \mathrm{P}_{2}, \mathrm{P}_{3}, \ldots . ., \mathrm{P}_{99}\right)$.

$\mathrm{P} 1=\mathrm{Li}+\mathrm{c}$.(N/100-facum_interv.Anteriores, fint $\left.\mathrm{P}_{1}\right)$, para datosagrupados.

Li: límite real inferior del intervalo que contiene al primer Percentil. C amplitud del intervalo 


\section{MEDIDAS DE DISPERSIÓN}

Las medidas de centralización proporcionan una información parcial de la distribución de la variable. El rango y los cuantiles proporcionan cierta información complementaria , sin embargo es necesario completar esa información con medidas relativas a las desviaciones de los datos respecto a las medidas de centralización.

1) La Varianza =

$$
\begin{aligned}
& v=\frac{\sum(X i-X m)^{2}}{N} \\
& v=\frac{\sum f i \cdot(X i-X m)^{2}}{N}
\end{aligned}
$$

para datosagrupados, Xi es la marca de clase.

Publicado en formato digital: Prof. Silvia Stela FERREYRA. ANÁLISIS EXPLORATORIO DE DATOS ESPACIALES. EStadística ESpacial. Resúmenes. ReVista Geográfica Digital. IGUNNE. Facultad de Humanidades. UNNE. Año 11. No 21. Enero-Junio 2014. ISSN 1668-5180. Resistencia, Chaco. En: 
Revista Geográfica Digital. I GUNNE. Facultad de Humanidades. UNNE, Año 11. № 21. Enero - J unio 2014. ISSN 1668-5180 Resistencia, Chaco

\section{2) La Desviación Típica}

para datos agrupados, Xi es la marca de clase.

$$
\begin{aligned}
& \sigma=\sqrt{\frac{\sum(X i-X m)^{2}}{N}} \\
& \sigma=\sqrt{\frac{\sum f i .(X i-X m)^{2}}{N}}
\end{aligned}
$$

\section{3) Respecto de la mediana: Desviación intercuartílica y rango intercuartílico:}

$$
\mathrm{C}=\left(\mathrm{Q}_{3}-\mathrm{Q}_{1}\right) / 2
$$

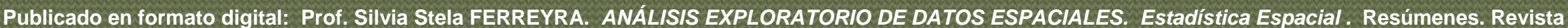
Geográfica Digital. IGUNNE. Facultad de Humanidades. UNNE. Año 11. No 21. Enero- Junio 2014. ISSN 1668-5180. Resistencia, Chaco. En: http:/hum.unne.edu.ar/revistas/geoweb/default.htm 
Cualquier distribución pueden ser caracterizados a partir de la media y la desviación típica. De esta forma se pueden comparar las diferencias de las distribuciones de una variable en dos espacios diferentes o la evolución de una misma variable en distintos momentos temporales.

* Si las distribuciones de dos series tienen igual media la que presente menor dispersión será la más conveniente( Ej.: se estudian las precipitaciones en dos zonas para escoger la más apropiada para un cultivo,

$\mathrm{Xm1}=43 \mathrm{~mm}$, Sxl= 12.59mm

$\mathrm{Xm} 2=43 \mathrm{~mm}, \mathrm{Sx} 2=6.75 \mathrm{~mm}$

Publicado en formato digital: Prof. Silvia Stela FERREYRA. ANÁLISIS EXPLORATORIO DE DATOS ESPACIALES. Estadística Espacial. Resúmenes. Revista Geográfica Digital. IGUNNE. Facultad de Humanidades. UNNE. Año 11. № 21. Enero - Junio 2014. ISSN 1668-5180. Resistencia, Chaco. En: 


\section{Coeficiente de variación de Pearson:}

\section{Expresa la homogeneidad de los datos, más o menos concentrados alrededor del promedio}

1.Cuando se quiere comparar la variabilidad de datos que tienen distintas unidades

2.Si las distribuciones de dos series tienen distinta media la comparación lo haremos por el coeficiente de variación.

$$
C V=\frac{\sum \sigma}{\bar{X}} \cdot 100
$$

Publicado en formato digital: Prof. Silvia Stela FERREYRA. ANÁLISIS EXPLORATORIO DE DATOS ESPACIALES. Estadística ESpacial. Resúmenes. Revista Geográfica Digital. IGUNNE. Facultad de Humanidades. UNNE. Año 11. No 21. Enero - Junio 2014. ISSN 1668-5180. Resistencia, Chaco. En: 


\section{MEDIDAS DE FORMA}

1) Para medir la asimetría de una distribución usamos unos parámetros que llamamos sesgo, cuyo valor nos da idea de la concentración de datos a un lado u otro de la media. $\mathrm{S}=(\mathrm{xm}-\mathrm{Mo}) / \mathrm{s}$ $\mathrm{S}>0$ sesgo derecho $\mathrm{S}=0$ simétrica $\mathrm{S}<0$ sesgo negativo

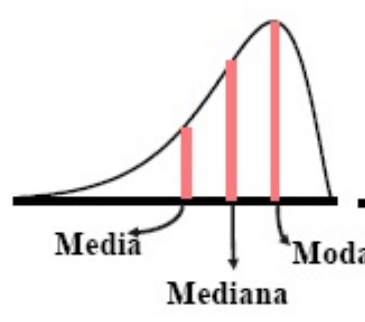

Asimétrica hacia la izquierda

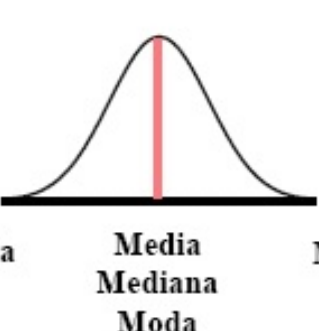

Moda Simétrica

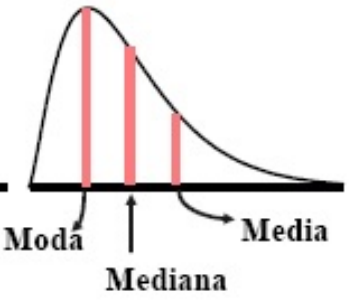

Asimétrica hacia la derecha

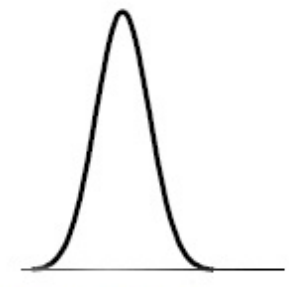

Leptocúrtica

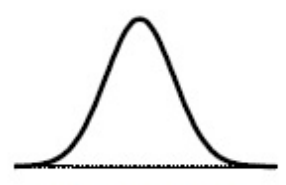

Mesocúrtica

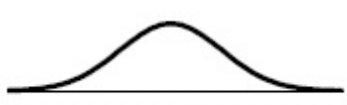

Platicúrtica
2) Para medir el grado de apuntamiento de la curva, si es muy aguda hacia arriba o muy aplanada.

$\mathrm{K}=\mathrm{Q} / \mathrm{P}_{90}-\mathrm{P}_{10}$, con $\mathrm{Q}=\left(\mathrm{Q}_{3}-\mathrm{Q}_{1}\right) / 2$

$\mathrm{K}>3$ Leptocúrtica

$\mathrm{K}=3$ Mesocúrtica

K<3 Platicúrtica

Publicado en formato digital: Prof. Silvia Stela FERREYRA. ANÁLISIS EXPLORATORIO DE DATOS ESPACIALES. Estadistica Espacial. Resúmenes. Revista Geográfica Digital. IGUNNE. Facultad de Humanidades. UNNE. Año 11. No 21. Enero - Junio 2014. ISSN 1668-5180. Resistencia, Chaco. En: http://hum.unne.edu.ar/revistas/geoweb/default.htm 
El análisis exploratorio de datos espaciales lo podemos realizar con la extensión Geostatical Analyst ( Analista Geoestadística), del programa de Arc Gis.

Los objetivos del análisis exploratorio en general son:

Examinar la distribución de datos $\rightarrow$ Distribución de frecuencias

* Detectar valores extremos (outliers) $\rightarrow$ Diagrama Box -Plot

*xaminar la correlación espacial entre variables

espaciales $\rightarrow$ Diagramas de Dispersión .

Examinar la autocorrelación espacial.

\&ntender la covariación entre múltiples variables.

Realizar transformaciones de los datos si son requeridas.

Publicado en formato digital: Prof. Silvia Stela FERREYRA. ANÁLISIS EXPLORATORIO DE DATOS ESPACIALES. Estadística Espacial. Resúmenes. Revista Geográfica Digital. IGUNNE. Facultad de Humanidades. UNNE. Año 11. № 21. Enero - Junio 2014. ISSN 1668-5180. Resistencia, Chaco. En:

http:I/hum.unne.edu.ar/revistas/geoweb/default.htm 
Revista Geográfica Digital. L GUNNE. Facultad de Humanidades. UNNE. Año 11. NNo 21. Enero - J unio 2014.

ISSN 1668-5,180-Resistencia, Chaco

\section{HISTOGRAMA}

\section{Su obtención lo hacemos mediante el ícono que lo caracteriza.}

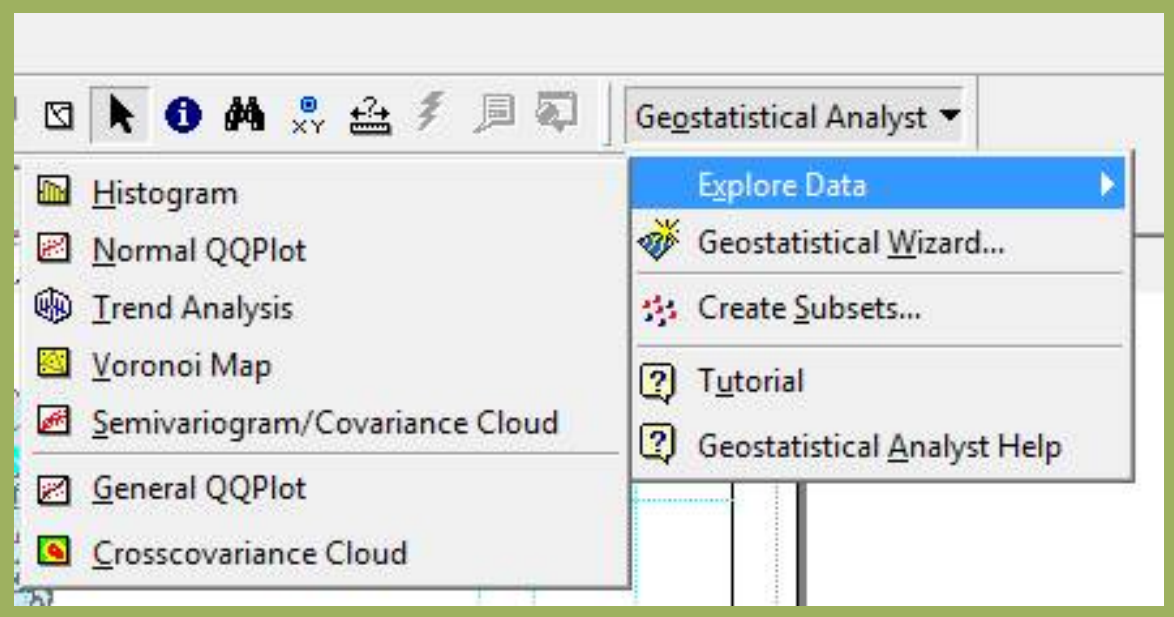

\section{Permite una descripción univariada de los datos.}

Publicado en formato digital: Prof. Silvia Stela FERREYRA. ANÁLISIS EXPLORATORIO DE DATOS ESPACIALES. Estadistica ESPacial. Resúmenes. Revista Geográfica Digital. IGUNNE. Facultad de Humanidades. UNNE. Año 11. № 21. Enero - Junio 2014. ISSN 1668-5180. Resistencia, Chaco. En: http:l/hum.unne.edu.ar/revistas/geoweb/default.htm 


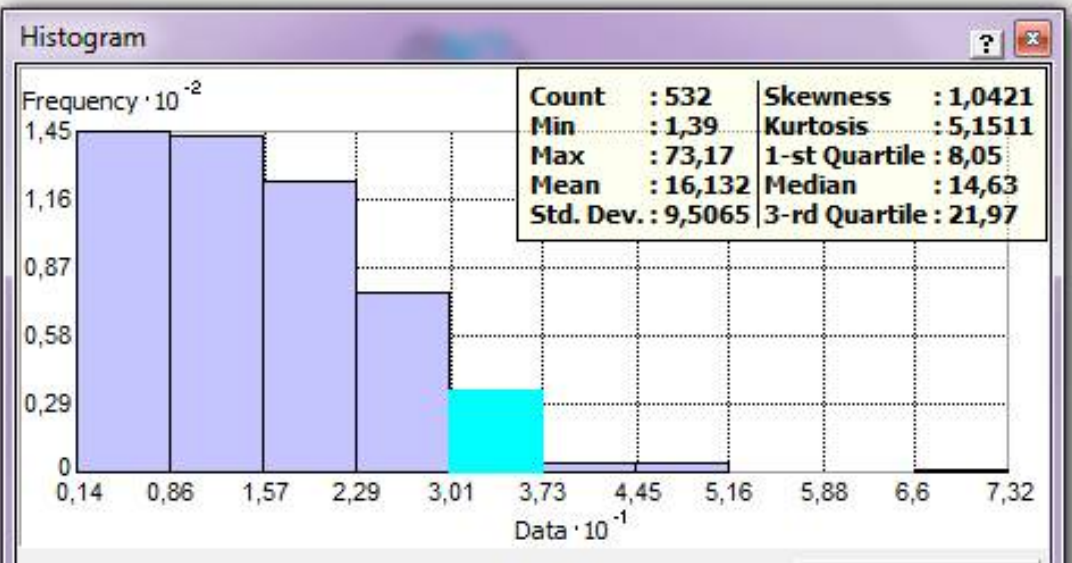

Tip: Click or drag over bars to select

Bars:

\section{$\sqrt{ }$ Statistics}

/ Transformation

Transformation: None $\quad$

$>$ Data Source

Layer:

deptos_arg_2001

Attribute:

- P_HgNBI01

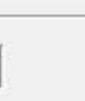

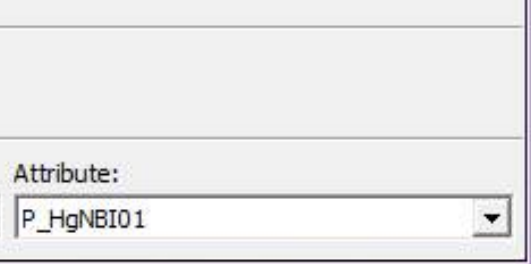

Presenta una distribución de frecuencias y un cuadro resumen de algunos estadísticos descriptivos de la centralidad de los datos, la dispersión y la forma.

La distribución de frecuencias se presenta mediante una serie de barras que agrupan los valores en un número de clases o intervalos. La altura de cada barra representa la frecuencia absoluta o relativa, en el ejemplo observamos la distribución de frecuencias del NBI de los departamentos de la Argentina.

Publicado en formato digital: Prof. Silvia Stela FERREYRA. ANÁISIS EXPLORATORIO DE DATOS ESPACIALES. Estadística Espacial. Resúmenes. Revista Geográfica Digital. IGUNNE. Facultad de Humanidades. UNNE. Año 11. № 21. Enero-Junio 2014. ISSN 1668-5180. Resistencia, Chaco. En: http:l/hum.unne.edu.ar/revistas/geoweb/default.htm 
Revista Geográfica Digital. I GUNNE. Facultad de Humanidades. UNNE..Año 11. № 21. Enero - J unio 2014. ISSN 1668-5180 Resistencia, Chaco

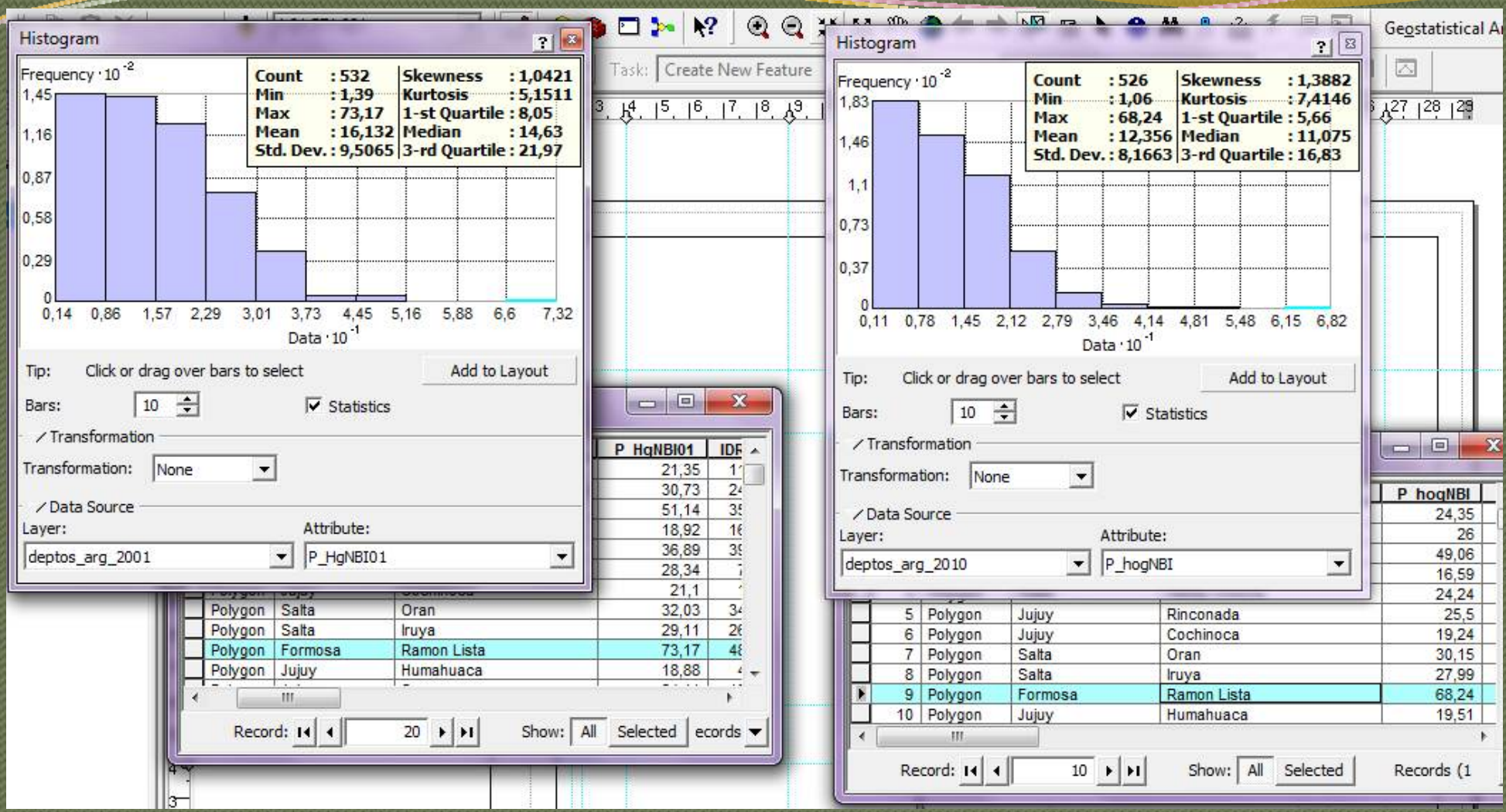

Las medidas de centralidad nos dan idea de donde está el centro y otras partes de la distribución.

La media es el promedio aritmético de los datos, da idea del centro de la distribución. La mediana representa una proporción acumulada del o,50, el 50\% de los datos está por debajo de ella y el otro 50\% por arriba, también da idea del centro de la distribución. El primer y tercer cuartil corresponden a una proporción acumulada del 0,25 y 0,75 respectivamente.

Publicado en formato digital: Prof. Silvia Stela FERREYRA: ANÁLISIS EXPLORATORIO DE DATOS ESPACIALES. Estadística Espacial. Resúmenes. Revista Geográfica Digital. IGUNNE. Facultad de Humanidades. UNNE. Año 11. № 21. Enero-Junio 2014. ISSN 1668-5180. Resistencia, Chaco. En: http:lihum.unne.edu.ar/revistas/geoweb/default.htm 
Como los datos están ordenados en forma creciente el 25\% de los datos están por debajo del primer cuartil y el 25\% de los datos están por encima del tercer cuartil.

- Las medidas de dispersión indican la mayor o menor agrupación de los datos alrededor del valor medio.

- La desviación típica que es la raíz cuadrada de la varianza (media del cuadrado de las desviaciones), describe la dispersión de los datos respecto a la media en las mismas unidades que los datos originales.

El hecho de que la media y mediana no coincidan y el coeficiente de asimetría esté lejos del o, evidencian la falta de normalidad de la variable. La cola derecha de la distribución indica la presencia de un punto con un NBI elevado. 


\section{GRAFICOS DE BARRA}

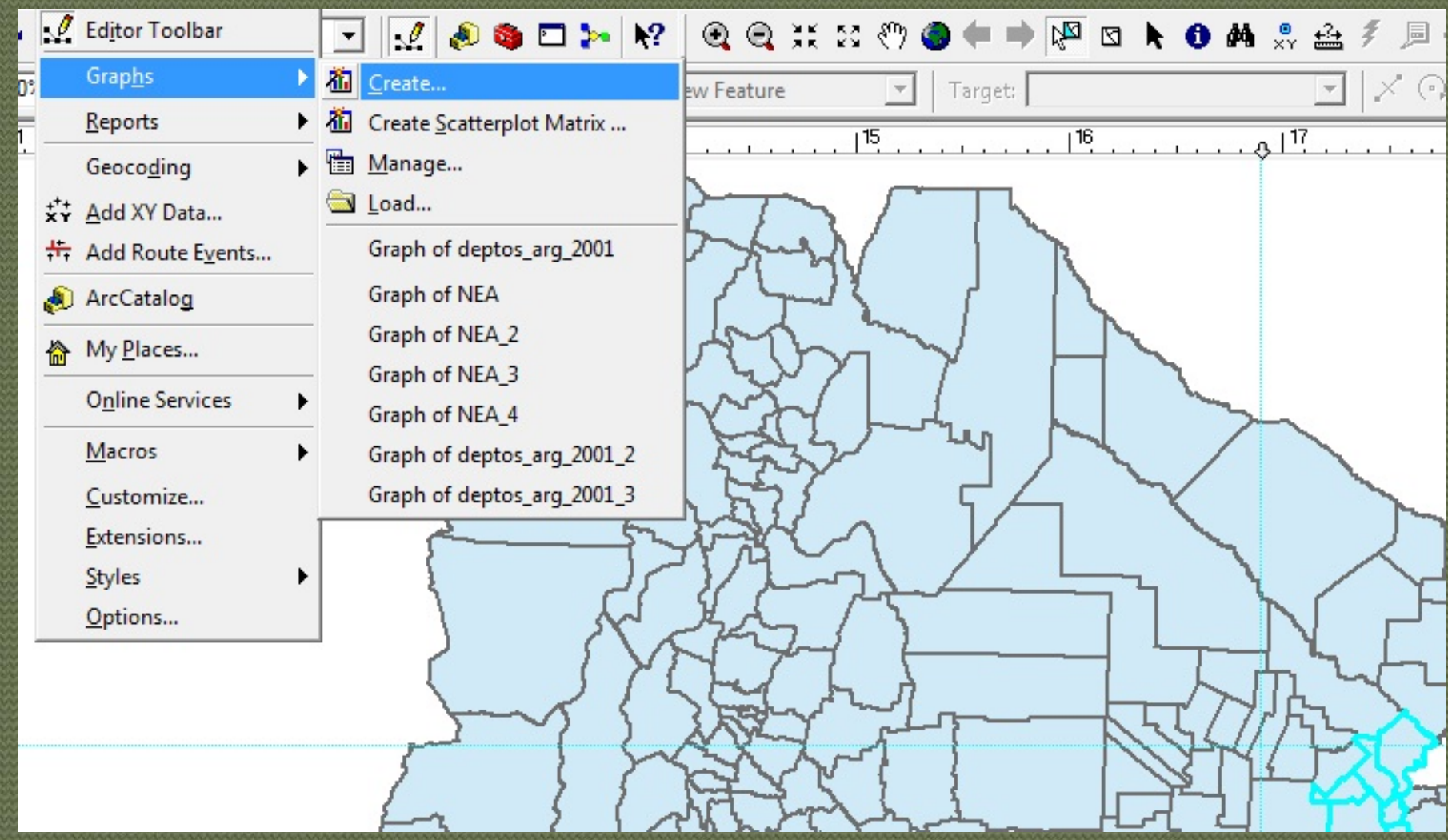

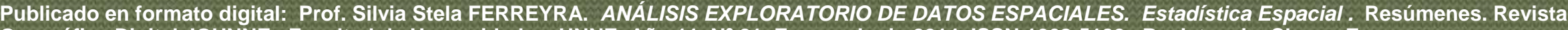
Geográfica Digital. IGUNNE. Facultad de Humanidades. UNNE. Año 11. No 21. Enero - Junio 2014. ISSN 1668-5180. Resistencia, Chaco. En:

http:I/hum.unne.edu.ar/revistas/geoweb/default.htm 


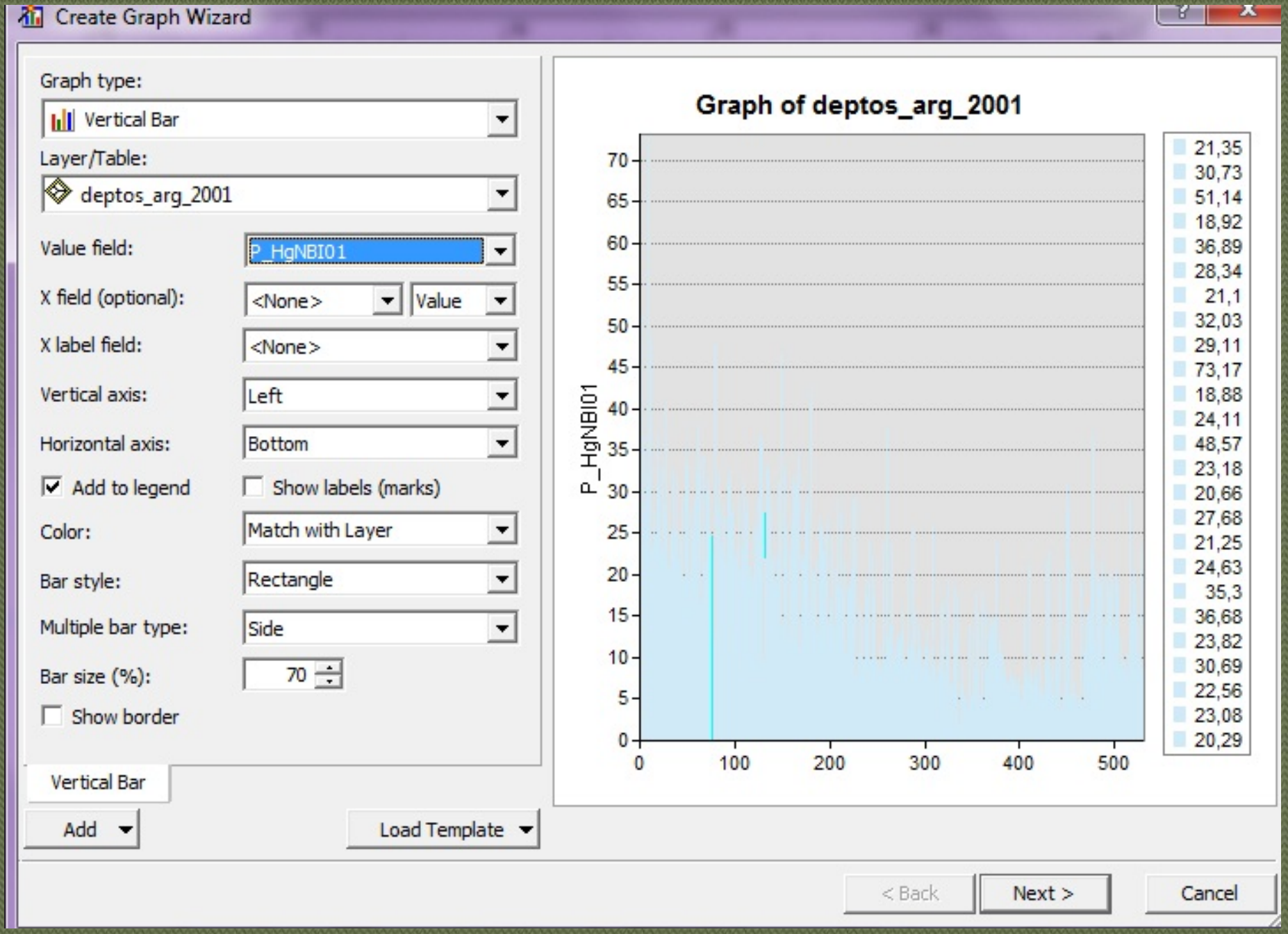

Publicado en formato digital: Prof. Silvia Stela FERREYRA. ANÁLISIS EXPLORATORIO DE DATOS ESPACIALES. Estadística Espacial. Resúmenes. Revista Geográfica Digital. IGUNNE. Facultad de Humanidades. UNNE. Año 11. No 21. Enero-Junio 2014. ISSN 1668-5180. Resistencia, Chaco. En: http:I/hum.unne.edu.ar/revistas/geoweb/default.htm 


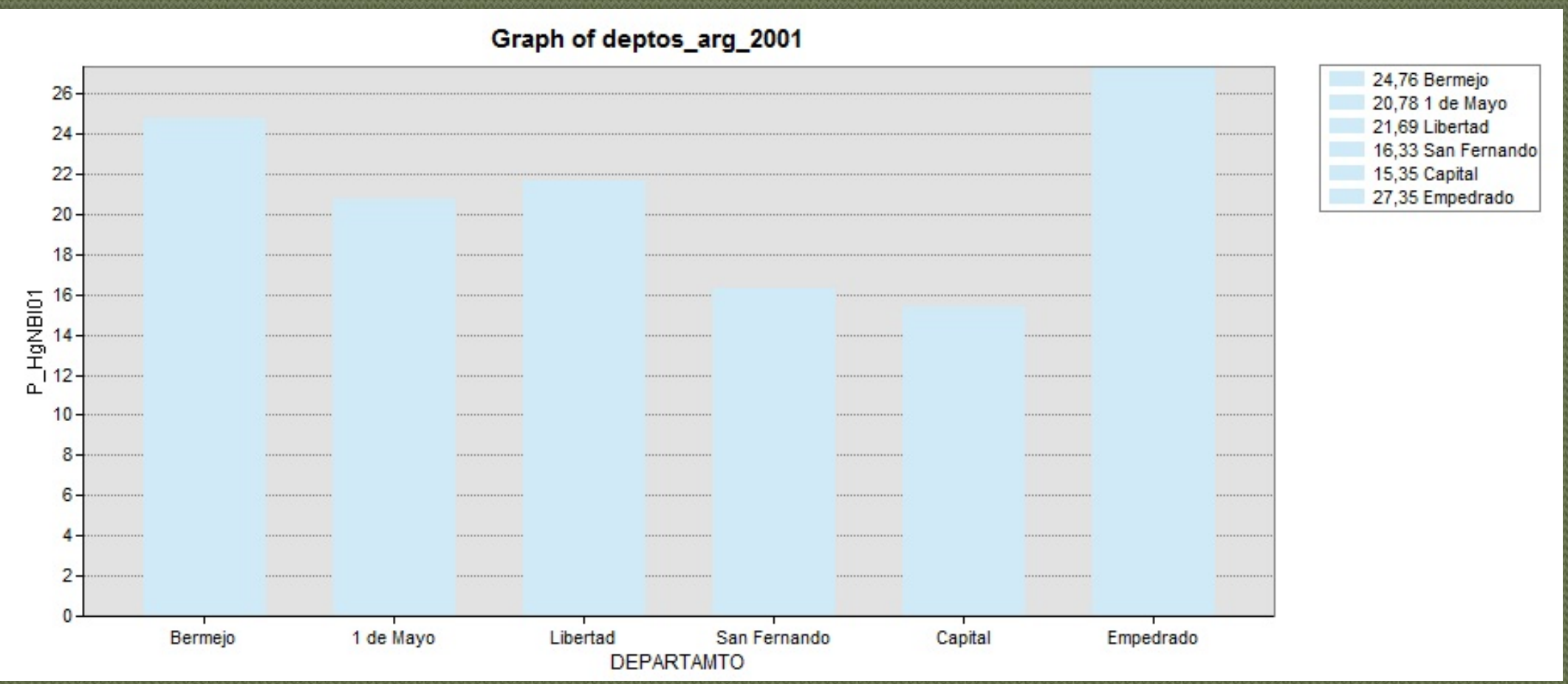

Publicado en formato digital: Prof. Silvia Stela FERREYRA. ANALISIS EXPLORATORIO DE DATOS ESPACIALES. Estadística Espacial. Resúmenes. Revista Geográfica Digital. IGUNNE. Facultad de Humanidades. UNNE. Año 11. No 21. Enero-Junio 2014. ISSN 1668-5180. Resistencia, Chaco. En: http:I/hum.unne.edu.ar/revistas/geoweb/default.htm 


\section{DIAGRAMA DE CAJAS O BOX PLOT}

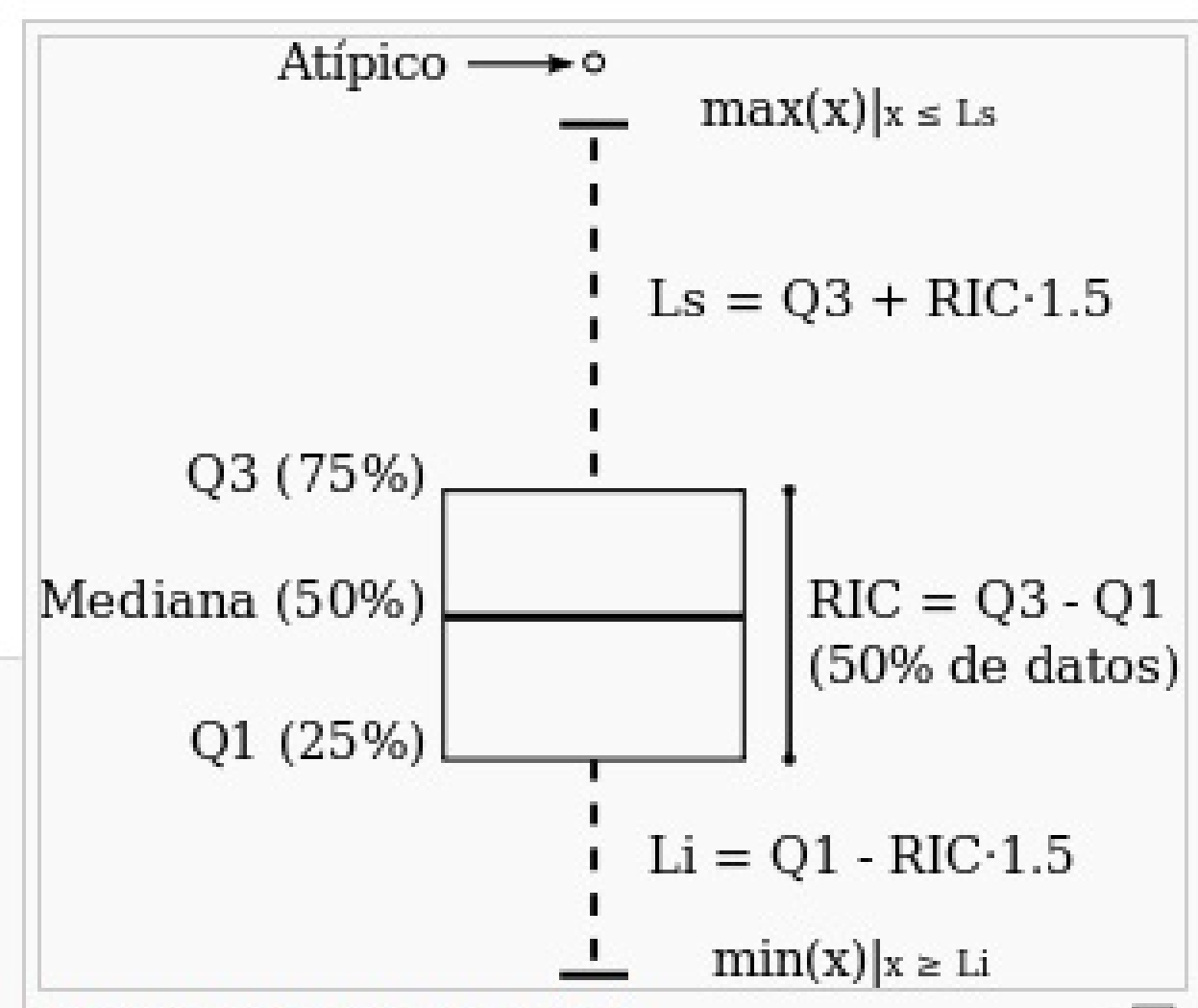

Diagrama de caja (Box-Plot).

Publicado en formato digital: Prof. Silvia Stela FERREYRA. ANÁLISIS EXPLORATORIO DE DATOS ESPACIALES. Estadística Espacial. Resúmenes. Revista Geográfica Digital. IGUNNE. Facultad de Humanidades. UNNE. Año 11. No 21. Enero - Junio 2014. ISSN 1668-5180. Resistencia, Chaco. En: http:l/hum.unne.edu.ar/revistas/geoweb/default.htm 
Un Diagrama de caja es un gráfico, basado en cuartiles, mediante el cual se visualiza un conjunto de datos. Está compuesto por un rectángulo, la "caja", y dos brazos, los "bigotes".

Es un gráfico que suministra información sobre los valores mínimo y máximo, los cuartiles $Q_{1}, Q_{2}$ o mediana y Q3, y sobre la existencia de valores atípicos y la simetría de la distribución.

Los bigotes, las líneas que se extienden desde la caja, se extienden hasta los valores máximo y mínimo de la serie o hasta 1.5 veces el RIC.

Cuando los datos se extienden más allá de esto, significa que hay valores atípicos en la serie y entonces hay que calcular los límites superior e inferior, Li y Ls.

Para ello, se consideran atípicos los valores inferiores a Q1-1.5*RIC= $5.66-1.5^{*}(16.83-5.66)=-11.095$

o superiores a $Q_{3}+1.5^{*} \mathrm{RIC}=16.83+1.5(16.83-5.66)=33.585$

Después se buscan los últimos valores que NO son atípicos, que serán los extremos de los bigotes. En el ejemplo NBI 2010: 1.06 y 33.33

Marcar como atípicos todos los datos que están fuera del intervalo (Li, Ls). En el ejemplo: 1.06 y 33.33

Además, se pueden considerar valores extremadamente atípicos aquellos que exceden $Q_{1-3}{ }^{*}$ RIC $=5.66-3^{*}(16.83-5.66)=-27.87$

o $\mathrm{Q}_{3}+3{ }^{*} \mathrm{RIC}=50.34$ 


\section{DIAGRAMA DE CAJAS O BOX PLOT}

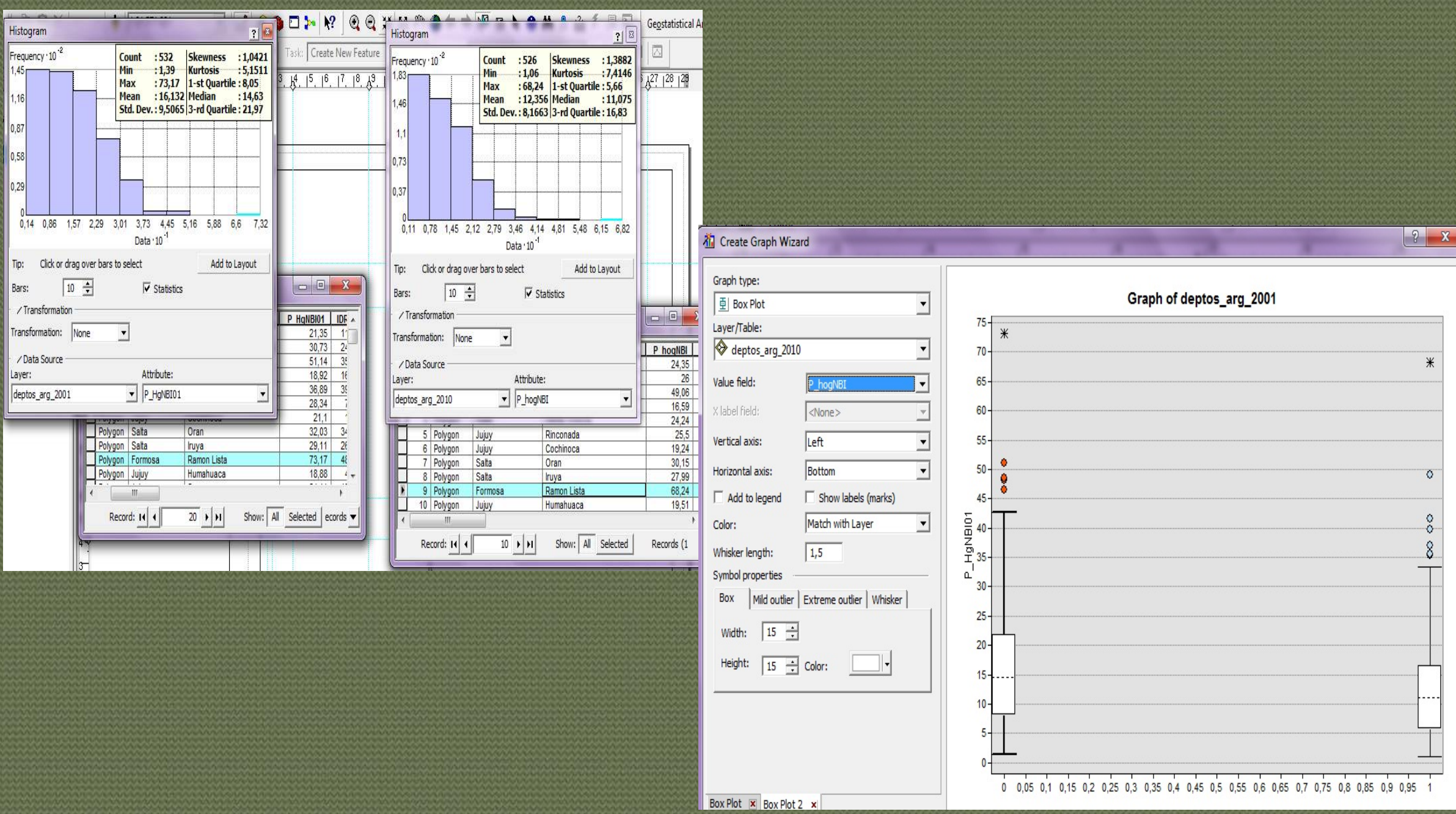




\section{GRAFICO CIRCULAR}

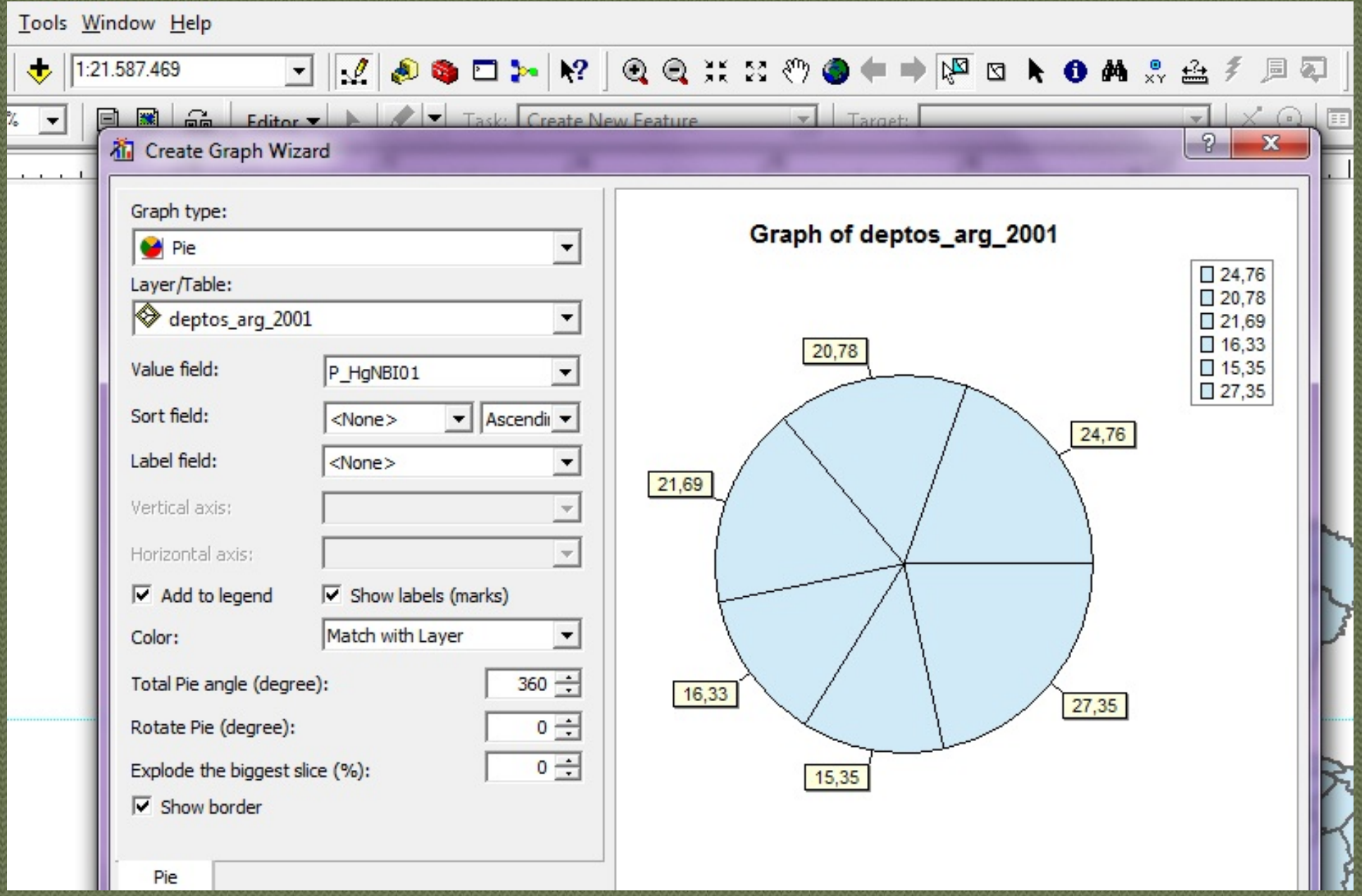

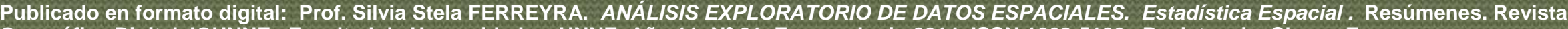
Geográfica Digital. IGUNNE. Facultad de Humanidades. UNNE. Año 11. No 21. Enero- Junio 2014. ISSN 1668-5180. Resistencia, Chaco. En:

http:I/hum.unne.edu.ar/revistas/geoweb/default.htm 


\section{DIAGRAMA DE DISPERSIÓN}

Diagrama de dispersión es la nube de puntos que resulta de la

representación gráfica bidimensional de las variables analizadas, donde cada punto tiene como abscisa -(Xi)- el valor de la variable que se considera independiente y como ordenada -(Yi)- el valor de la variable considerada dependiente.

$$
r=\frac{\sum(X i-\bar{X}) \cdot(Y i-\bar{Y})}{\sqrt{\sum(X i-\bar{X})^{2}} \cdot \sqrt{\sum(Y i-\bar{Y})^{2}}}
$$

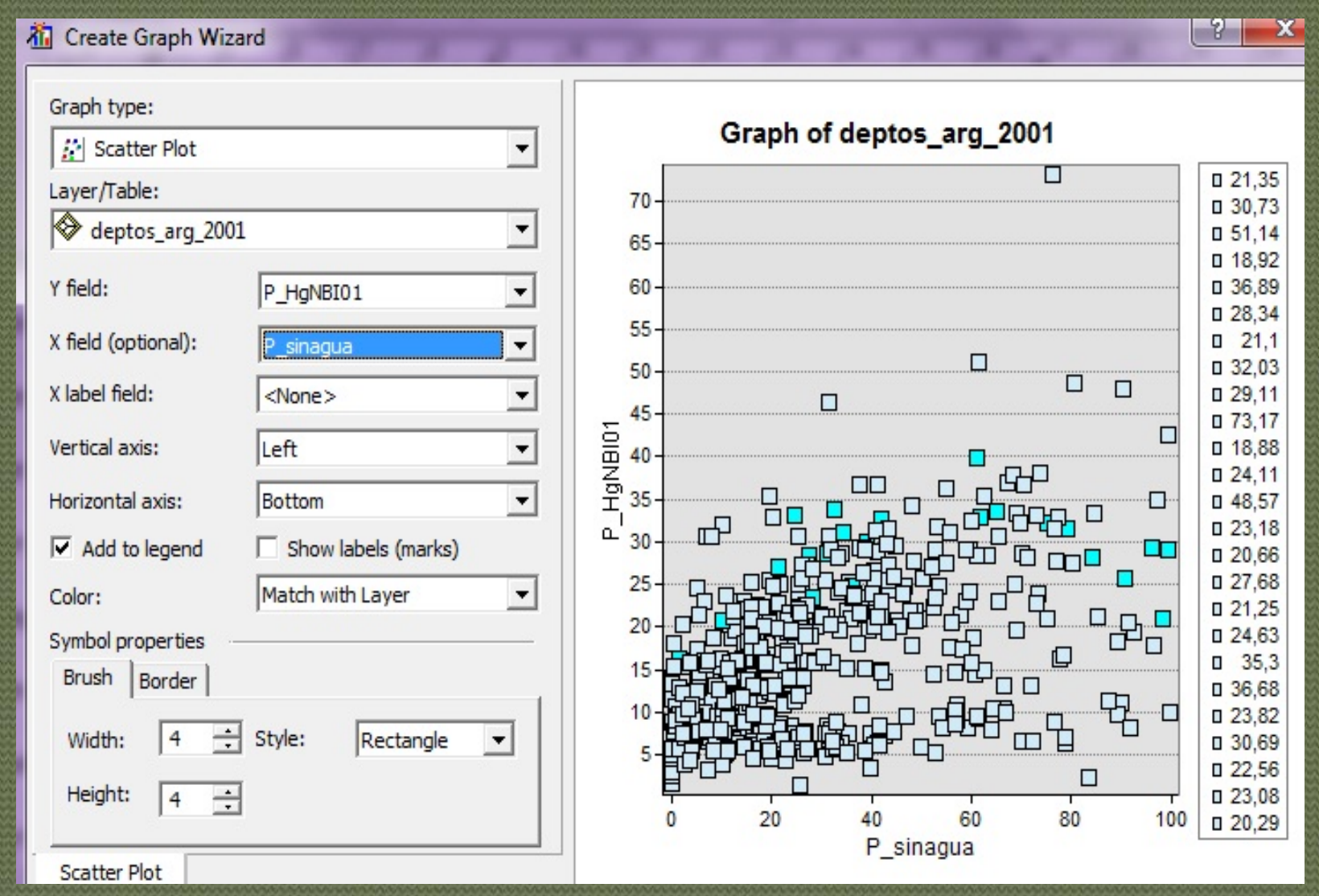




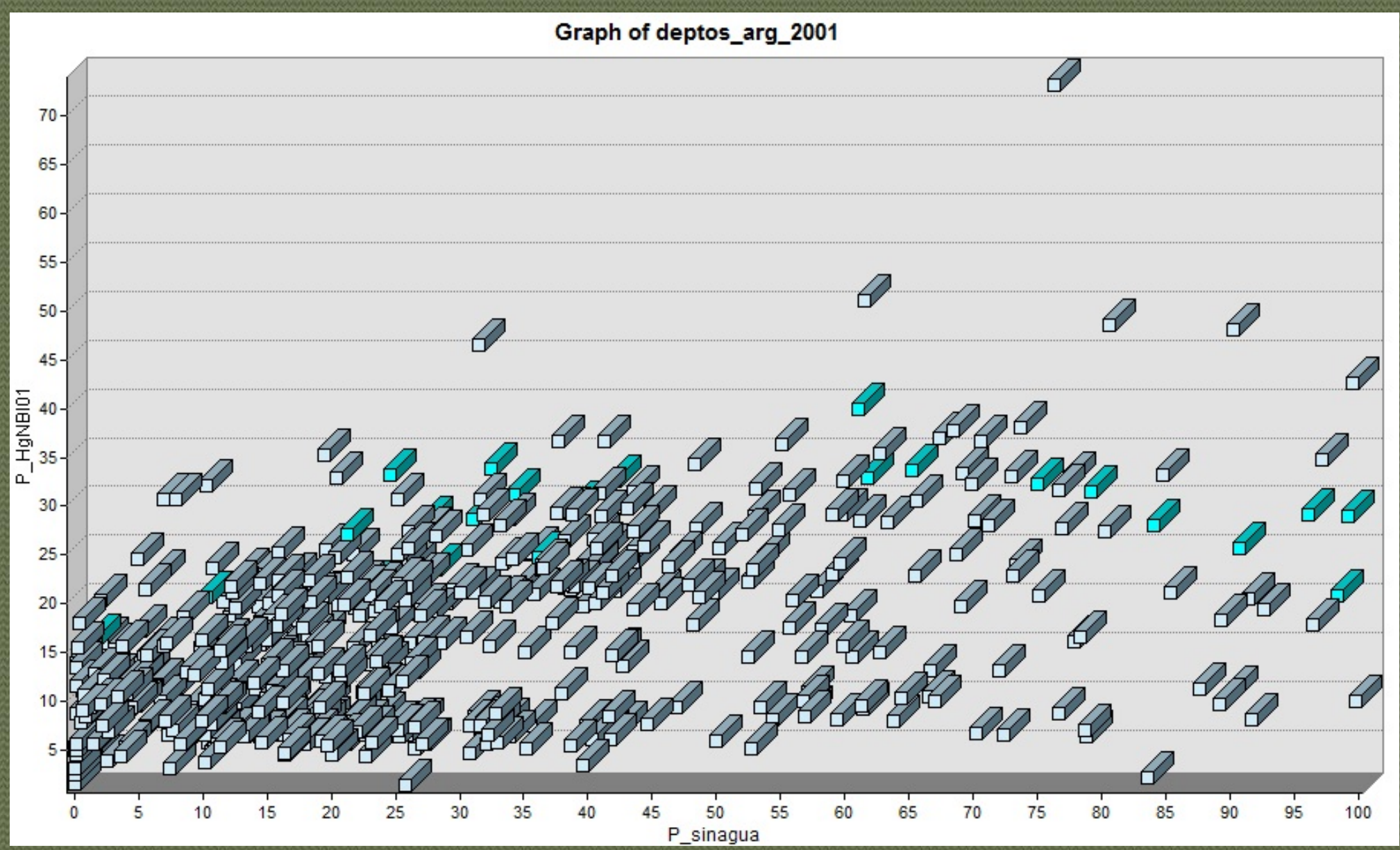

Publicado en formato digital: Prof. Silvia Stela FERREYRA. ANÁLISIS EXPLORATORIO DE DATOS ESPACIALES. Estadística Espacial. Resúmenes. Revista Geográfica Digital. IGUNNE. Facultad de Humanidades. UNNE. Año 11. No 21. Enero - Junio 2014. ISSN 1668-5180. Resistencia, Chaco. En:

http:I/hum.unne.edu.ar/revistas/geoweb/default.htm 


\section{BIBLIOGRAFIA}

Módulos II. Exploración de datos y Estadística Espacial. Unidad 4. Conceptos de estadística. Curso e- learning de Cartografía temática avanzada. Ministerio de Fomento. Dirección General del Instituto Geográfico Nacional. Módulos III y IV del Curso de Posgrado en estadística aplicada a la Investigación de la UNC.

Sistemas y Análisis de la Información Geográfica. Unidad 10.1. Análisis Exploratorio de Datos Espaciales. M.R. Cañada Torrecilla

Nociones de Estadística y Probabilidad, Nélida Chica de Galassi -Analía Piccini - E. Rossi de Greco y Lilián Chica de Bonnet.

Publicado en formato digital: Prof. Silvia Stela FERREYRA. ANÁLISIS EXPLORATORIO DE DATOS ESPACIALES. Estadística Espacial. Resúmenes. Revista Geográfica Digital. IGUNNE. Facultad de Humanidades. UNNE. Año 11. No 21. Enero - Junio 2014. ISSN 1668-5180. Resistencia, Chaco. En: http:llhum.unne.edu.ar/revistas/geoweb/default.htm 